\title{
Simultaneous Bony Mallet Fingers of Two Phalanx in a Patient: A Rarely Seen Case Report
}

\author{
Erdal Uzun ${ }^{1}$ \\ ${ }^{1}$ Orthopedic and Traumatology Clinic, Kayseri Research and Training Hospital, Kayseri, Turkey. \\ Received: 25 April 2016 Accepted: 15 June 2016, Published online: 25 April 2017 \\ (C) Ordu University Institute of Health Sciences, Turkey, 2017
}

\begin{abstract}
Mallet finger deformity is a common hand injury and usually occurs after a direct impact of sudden and extreme trauma forcing the distal interphalangeal joint to get flexion with an extended proximal interphalangeal joint. Deformity is especially associated with ball use disrupting the extensor mechanism at the base of distal phalanx. Mallet finger deformity in acute stage can be treated by immobilization of distal interphalangeal joint with hyperextension splints. But the surgery becomes the choice in delayed cases and if there is a fracture including more than one third of articular surface of distal interphalangeal joint or distal phalanx volar subluxation. In the literature there are great sum of studies including mallet finger deformities as simultaneous, bilateral or multiple variations and treatment strategies. But in the literautre there is no study about simultaneous bony mallet fingers deformity reported. Here, we present the case of a 30 year old female with simultaneous bony mallet fingers in the third and fourth ray and the conservative treatment of with splinting for six weeks. At the last time follow up the patient was able to use her hand without any diffuculty and she had full range of motion of distal interphalangel joint.
\end{abstract}

Key words: Bony mallet finger, Simultaneous, Treatment, Conservative

Address for correspondence/reprints:

Erdal Uzun

Telephone number: +90 (352) 3368884

E-mail: nuzuladre@gmail.com

DOI: $10.19127 / \mathrm{mbsjohs.309375}$

\section{Introduction}

Mallet finger is a common fingertip injury with a prevalence of $1: 10,000$ that involves disruption of the extensor mechanism at its terminal insertion, resulting in inability to actively extend the distal interphalangeal joint (Bloom et al., 2013). The injury is most commonly sustained during sporting activity activity especially associated with ball use (Er et al., 2014). The dominant hand middle, ring, and little fingers are most often affected (Bloom et al., 2013). If the mallet deformity is not treated appropriately it can lead to permanent deformities like flexion deformity and inability to actively extend the distal interphalangeal (DIP) joint and swan neck deformity (Kanaya et al., 2013). Surgery is reserved in delayed cases and if there is a fracture including more than one third of articular surface of distal interphalangeal joint, distal phalanx volar subluxation, conservative management failure, avulsed fragment that fails 
reduction, and absence of full passive extension of the joint (Anderson, 2011). In the literature there are great sum of studies including mallet finger deformities as simultaneous, bilateral or multiple variations and treatment strategies (Bloom et al., 2013; Er et al., 2013; Kanaya et al., 2013; Miranda et al., 2015). But simultaneous bony mallet fingers deformity is not reported. Here, we present the case of a 30 year old female with simultaneous bony mallet fingers in the third and fourth ray and our treatment strategy.

\section{Case Report}

A 30-year-old healthy female admitted to our outpatient clinic with a traumatic injury that had occured while playing volleyball one day before. She gave the history of the ball hit of her dominant right hand both the middle and ring digits on the outstretched position. She had no personal or family history of previous mallet fingers. On physical examination her third and ring fingers were both in flexion position. She was unable to extend her right DIP joint of the third and ring fingers and she had the complaint of extreme pain and deformity on both DIP joints. The mallet deformity of both fingers were obvious at physical examination and the diagnose was confirmed radiologically. There was bony mallet of the third and ring fingers on the direct rontgenogram without subluxation (Figure). On the lateral radiographs it could be seen that avulsion fractures identified were containing less than one third of DIP joint surface. So we considered conservative treatment with application of splinting both the third and ring fingers' DIP joints in extention position for 6 weeks period. After 3 months at the last follow-up a full recovery was achieved for both fingers.

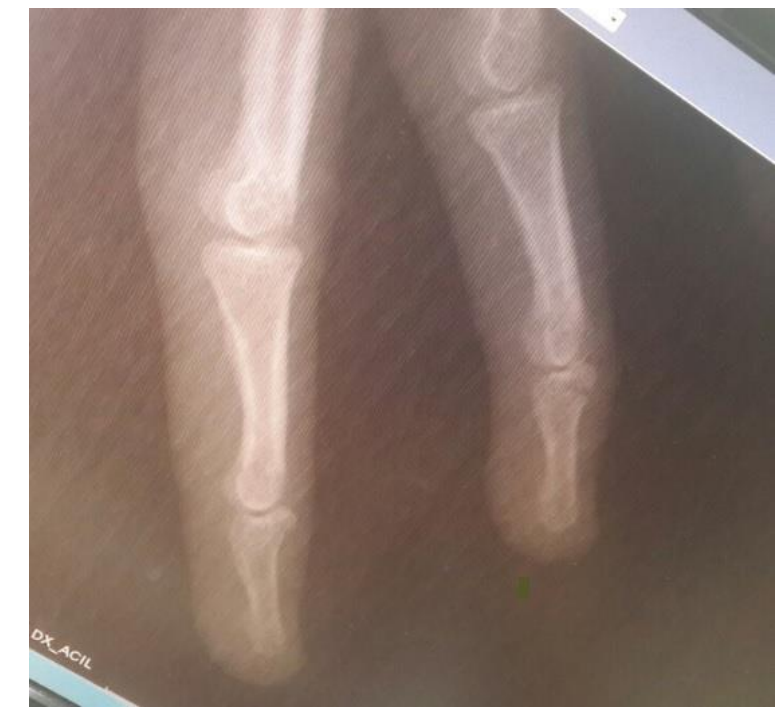

Figure: There was bony mallet of the third and ring fingers on the direct rontgenogram without subluxation.

\section{Discussion}

Avulsion injuries are most commonly sustained during sporting activity (Çıraklı et al., 2013). Mallet finger is one of avulsion injuries and a common fingertip injury with a prevalence of 1:10,000 (Bloom et al., 2013). The dominant hand middle, ring, and little fingers are most often affected (Bloom et al., 2013). Deformity occurs disrupting the extensor mechanism with or without bony fragment of distal phalanx. Mallet finger deformity in acute stage can be treated by immobilization of distal interphalangeal joint with hyperextension splints. Surgery is reserved in delayed cases and if there is a fracture including more than one third of articular surface of distal interphalangeal joint, distal phalanx vo $\neg$ lar subluxation, conservative management failure, avulsed fragment that fails reduction, and absence of full passive extension of the joint (Anderson, 2011). There are different techniques reported for bony mallet deformities as extansor bloc pinnig, percutaneous blunt needle reduction, internal fixation using hook plate and screws (Er et al., 2014; Miranda et al., 2015). But some devastating complications can occur performing surgical strategy such as pin-site infections and potentially osteomyelitis. Stern et al. suggested that outcome of conservative treatment by splinting for a minimum of six weeks is equal to operative treatment and has a significantly lower complication rate (Stern and Kastrup, 1988). Similarly Hamas et al. supported the idea that bony injury can be treated conservatively with splinting 
of the distal phalanx in extension for 6-8 weeks where the bony fragments appear to afford better tendon healing (Hamas et al., 1978). The patient in this report had bony simultaneous mallet fingers of both the middle and ring digits with containing less than one third of DIP joint surface. So we considered splinting instead of surgical treatment. At the last follow-up a full recovery was achieved for both fingers reducing surgical complication riscs.

Mallet finger deformity is offen seen in a single digit, but simultaneous mallet deformity is quite rare. In the literature we couldn't see any study about simultaneous bony mallet deformity of the dominant hand, to our opinion this is the first study reported.

In conclusion, where there is no definitive evidence that outcomes are better with anatomical reduction of mallet deformities, conservative treatment may be a choice also for simultaneous bony mallet deformities with reducing surgical complication riscs. And also patients must be more careful with the ball use where it threatens not only the sportsman particularly prone to hand injuries but also the peaple have no personal risc factor or familial history.

Informed Consent: Necessary information using the patient information form and consent form was taken from the patients

Peer-review: Externally peer-reviewed.

Author Contributions: Concept- EU, DesignEU, Supervision EU, Funding- EU, Materials- EU, Data Collection and/or Processing- EU, Analysis and/or Interpretation- EU, Literature Review- EU, Writing- EU, Critical Review- EU.

Conflict of Interest: No conflict of interest was declared by the author.

Financial Disclosure: The author declared that this study hasn't received any financial support.

\section{References}

Anderson D. Mallet finger-Management and patient compli-ance. Aust Fam Physician 2011;40:47-8.

Bloom JM, Khouri JS, Hammert WC. Current concepts in the evaluation and treatment of mallet finger injury. Plast Reconstr Surg 2013;132:560-6.

Çıraklı A, Zengin EÇ, Sezgin H, Çıraklı S, Erdoğan M. Avulsion fracture of the anterior inferior iliac spine among amateur soccer players. JAEMCR 2013;4:136-8.

Er MS, Erten RA, Eroğlu M, Altınel L. Bilateral simultaneous mallet finger: An unusual case. Dicle Medical Journal 2014;41(1):231-3.

Hamas RS, Horrell ED, Pierret GP. Treatment of mallet fi nger due to intra-articular fracture of the distal phalanx. J Hand Surg Am 1978;3:361-3.

Kanaya K, Wada T, Yamashita T. The Thompson proce $\neg$ dure for chronic mallet finger deformity. J Hand Surg Am 2013;38:1295-1300.

Miranda BH, Murugesan L, Grobbelaar AO, Jemec B. Percutaneous Blunt Needle Reduction of Bony Mallet Injuries. Tech Hand Up Extrem Surg 2015;19(2):81-3.

Stern PJ, Kastrup JJ. Complications and prognosis of treatment of mallet finger, J Hand Surg 1988;13:329-34. 